\title{
OPTIMUM DESIGN OF FIBER ANGLE AND HOLE ORIENTATION OF AN ORTHOTROPIC PLATE
}

\author{
Xiaoli Zhang, Aizhong Lu, Shaojie Wang, Ning Zhang \\ North China Electric Power University, Institute of Hydroelectric and Geotechnical Engineering, Beijing, China \\ e-mail: zning1125@ncepu.edu.cn (N. Zhang)
}

\begin{abstract}
With the goal of decreasing the stress concentration along the hole boundary in an orthotropic plate under inequi-biaxial loadings, an optimum design of the fiber angle and hole orientation is presented. The maximum absolute tangential stress along the hole boundary is taken as the objective function, and the fiber orientation angle and the hole orientation angle are considered as design variables. The conformal transformation method of a complex function and the Differential Evolution (DE) algorithm are used. Two non-circular shapes, ellipse and hexagon are taken as examples to analyze the problem. Based on the results, we can conclude that the major axis of elliptical holes should be designed in the direction of the maximum external loading for a perforated structure in an orthotropic plate. However, the principal direction that has the larger Young's modulus should be inclined to the direction of the minimum loading, especially for a significantly orthotropic plate.
\end{abstract}

Keywords: orthotropic plate, fiber orientation angle, hole orientation angle, conformal transformation method, differential evolution algorithm

\section{Introduction}

Natural materials, such as wood and rock, and composite materials, such as fiberboard, epoxy resin bonded fiber and fiber reinforced polymer, all display anisotropic properties. Specifically, orthotropic plates with different holes have found widespread applications in various fields such as aerospace, marine, automobile and mechanics because of high specific stiffness and specific strength as well as the designability of the properties (Jain, 2009; Romeo, 2001; Li and Zheng, 2007). The stress concentration along the boundary of the hole becomes an important research problem for this structure (Toubal et al., 2005; Sharma, 2011; Engels et al., 2001). Generally, tangential stresses at different points along the hole boundary in orthotropic plates are different and, usually, it is the maximum value that depends the stability on the plate (Savin, 1961). Thus, it is important to decrease the tangential stress concentration along the hole boundary in the design of orthotropic plates.

Some researchers have studied the shape optimization of holes in isotropic plates based on different optimization criteria or methods. Bjorkman and Richards $(1976,1979)$ proposed the concept of a harmonic hole and obtained the optimal shape of the hole under different loads. Taking the minimum integration of the square of tangential stress as the objective, Dhir (1981) solved a series of shape optimization problems by the complex variables function, the same method as Bjorkman. Aiming at minimizing the maximum value of the tangential stress along the hole boundary, Lu et al. $(2014 \mathrm{a}, \mathrm{b})$ obtained the optimum shape of the support section of a tunnel at great depths using the complex variables function method. The hole-shape optimization problem has also been solved using the Evolutionary Structural Optimization (ESO) procedure (Ren et al., 2005) and the Simulated Annealing (SA) procedure (Sobótka et al., 2013).

So far, the existing studies have mainly focused on the shape optimization of hole in isotropic plates to decrease stress concentration. For many practical applications, however, the shape 
of the hole is always determined by some specific demands. Hence, the optimization results sometimes cannot meet the actual requirement. Furthermore, many plates in actual applications are orthotropic. For orthotropic plates, however, the determination of stress distribution is more complex than that for isotropic plates. Lekhnitskii $(1968,1981)$ used the complex function method, developed by Muskhelishvili (1963), to determine the stress in an anisotropic plate, and gave an accurate solution of stress around an elliptic hole under in-plane loading at infinity. Some researchers have obtained analytical solutions for other non-elliptic holes (Romeo, 2001; Rao et al., 2010; Daoust and Hoa, 1991; Ukadgaonker and Kakhandki, 2005; Rezaeepazhand and Jafari, 2008). Lu et al. (2015) found an accurate analytic solution of stress for plates with an arbitrarily shaped hole using the power-series method.

It can be seen from the stress analysis of an orthotropic plate that the stress distribution along the hole boundary mainly depends on the fiber orientation angle and the hole orientation angle for certain loadings and hole shapes. Therefore, how to decide the two angles of an orthotropic plate in minimization of the stress concentration is important. Sharma et al. (2014) researched the optimization of fiber orientation angle for single lamina of composite materials (graphite/epoxy and glass/epoxy) with circular and elliptical holes; however, the hole orientation angle was not taken into consideration. In this paper, not only the fiber orientation angle but also the hole orientation angle are taken into consideration, and other more complicated shapes of holes are considered, in which the maximum absolute value of the tangential stress reaches its minimum value.

Herein, the Differential Evolution (DE) algorithm (Storn and Price, 1997) is used, and the following assumptions are made: the orthotropic plate is infinite and elastic under the in-plane loading at infinity (see Fig. 1); the problem can be treated as a plane stress problem.

\section{Fundamental theories}

Although the optimization of fiber orientation angle and hole orientation of an orthotropic plate is an inverse problem, the process of obtaining the optimal results requires solving a series of forward problems. In every optimization process, the solution for the tangential stress along the hole boundary should be based on the material properties, external loads, shapes and orientation angles of the hole. In this paper, the conformal transformation method of the complex function is adopted to first map the outer regions of the hole in the physical plane to the outer regions of the unit circle in the image plane. Then, the analytical solution of stress along the hole boundary can be obtained using the power-series method.

\subsection{Transformation of the mapping function under different coordinates}

Figure 1 illustrates an orthotropic plate with an arbitrarily shaped hole under an in-plane loading. xoy is the global Cartesian coordinate system, where $x$ and $y$ are along the directions of $\sigma_{x}^{\infty}$ and $\sigma_{y}^{\infty}$, respectively. $x_{1} o y_{1}$ and $x^{\prime} o y^{\prime}$ are two local Cartesian coordinates. $x_{1}$ is the symmetric axis of the hole, and the angle $\alpha$ between the positive direction of the $x$-axis and $x_{1}$-axis denotes the hole orientation. $x^{\prime}$ and $y^{\prime}$ are along the principal direction of the elastic materials, and the angle $\varphi$ denotes the fiber orientation. The outer region of the hole in the local coordinates $x_{1} o_{1}$ ( $z$-plane) is mapped to the outer region of the unit circle ( $\zeta$-plane) using the following mapping function

$$
z_{*}=x_{1}+\mathrm{i} y_{1}=R_{1}\left(\zeta+\sum_{k=1}^{n} C_{k} \zeta^{-k}\right)
$$

where $\mathrm{i}=\sqrt{-1}$, and $R_{1}$ is a real constant and $C_{k}$ are complex constants denoting the size and shape of the hole, respectively. $\zeta=\rho \mathrm{e}^{\mathrm{i} \theta}(\theta$ is the polar angle in the $\zeta$-plane, $\rho=1$ on the hole 
boundary). When $n$ is large enough, Eq. (2.1) can describe a variety of shapes of the hole. The mapping function in the global coordinates should be obtained using the following method due to the change of the hole orientation angle.

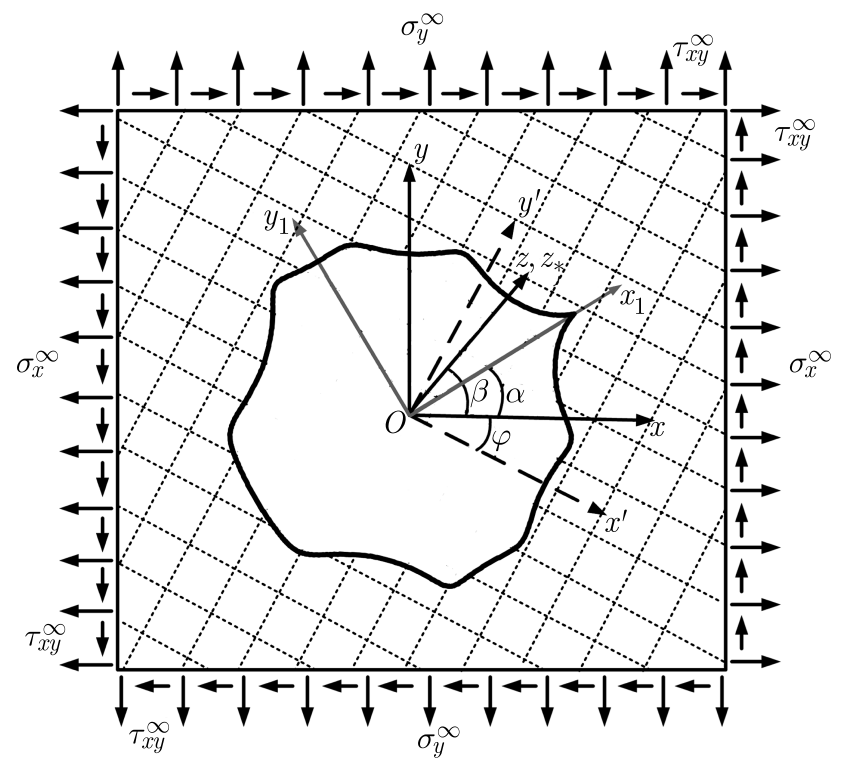

Fig. 1. Orthotropic plate with an arbitrarily shaped hole under an in-plane loading

As shown in Fig. 1, the local coordinates $x_{1} o y_{1}$ overlap the global coordinates xoy after rotating $\alpha$ degrees clockwise. The vector $z(z=x+\mathrm{i} y)$ with a length of $r$ in the coordinates xoy makes an angle $\beta$ with the $x$-axis.

In the global coordinates xoy

$$
z=r \mathrm{e}^{\mathrm{i} \beta}
$$

In the local coordinates $x_{1} o y_{1}$

$$
z_{*}=r \mathrm{e}^{\mathrm{i}(\beta-\alpha)}
$$

Then, Eq. (2.4) can be obtained because Eqs. (2.2) and (2.3) share the same vector $z$

$$
z_{*}=r \mathrm{e}^{\mathrm{i}(\beta-\alpha)}=\mathrm{e}^{-\mathrm{i} \alpha} r \mathrm{e}^{\mathrm{i} \beta}=\mathrm{e}^{-\mathrm{i} \alpha} z
$$

Then

$$
z=\omega(\zeta)=\mathrm{e}^{\mathrm{i} \alpha} z_{*}=\mathrm{e}^{\mathrm{i} \alpha} R_{1}\left(\zeta+\sum_{k=1}^{n} C_{k} \zeta^{-k}\right)=R\left(\zeta+\sum_{k=1}^{n} C_{k} \zeta^{-k}\right)
$$

It can be seen that the mapping function in the coordinates xoy can be easily determined by Eq. (2.5) as long as the corresponding mapping function in the coordinates $x_{1} o y_{1}$ is given even if the hole orientation angle $\alpha$ changes. Changing the real constant $R_{1}$ in Eq. (2.1) to a complex constant $R\left(R=R_{1} \mathrm{e}^{\mathrm{i} \alpha}\right)$ is what we need to do.

\subsection{The affine transformation and mapping}

The three complex variables involved in solving the anisotropic plane problems are $z, z_{1}$ and $z_{2}$, and the affine mathematical relationships between the three complex variables are

$$
\begin{aligned}
& z_{1}=x+\mu_{1} y=\gamma_{1} z+\delta_{1} \bar{z} \\
& z_{2}=x+\mu_{2} y=\gamma_{2} z+\delta_{2} \bar{z}
\end{aligned}
$$


where $\gamma_{1}=\left(1-\mathrm{i} \mu_{1}\right) / 2, \gamma_{2}=\left(1-\mathrm{i} \mu_{2}\right) / 2, \delta_{1}=\left(1+\mathrm{i} \mu_{1}\right) / 2, \delta_{2}=\left(1+\mathrm{i} \mu_{2}\right) / 2, \mu_{1}=\alpha_{1}+\mathrm{i} \beta_{1}$, $\mu_{2}=\alpha_{2}+\mathrm{i} \beta_{2}, \alpha_{k}$ and $\beta_{k}(k=1,2)$ are real constants related to the material properties.

If three polar coordinates are introduced, and by transforming the outer regions of the hole in the $z_{-}^{-}, z_{1^{-}}$, and $z_{2}$-planes into the outer regions of the unit circle in the $\zeta_{-}, \zeta_{1^{-}}$, and $\zeta_{2}$-planes, respectively, the three mapping functions which satisfy Eqs. (2.6) can then be obtained. The three mapping functions are expressed by $z=\omega(\zeta), z_{1}=\omega_{1}\left(\zeta_{1}\right)$ and $z_{2}=\omega_{2}\left(\zeta_{2}\right)$, respectively (Lu et al., 2015)

$$
\begin{aligned}
& z_{1}=\omega_{1}\left(\zeta_{1}\right)=\gamma_{1} R\left(\zeta_{1}+\sum_{k=1}^{n} C_{k} \zeta_{1}^{-k}\right)+\delta_{1} \bar{R}\left(\frac{1}{\zeta_{1}}+\sum_{k=1}^{n} \bar{C}_{k} \zeta_{1}^{k}\right) \\
& z_{2}=\omega_{2}\left(\zeta_{2}\right)=\gamma_{2} R\left(\zeta_{2}+\sum_{k=1}^{n} C_{k} \zeta_{2}^{-k}\right)+\delta_{2} \bar{R}\left(\frac{1}{\zeta_{2}}+\sum_{k=1}^{n} \bar{C}_{k} \zeta_{2}^{k}\right)
\end{aligned}
$$

where $\zeta_{1}=\rho_{1} \mathrm{e}^{\mathrm{i} \theta_{1}}$ and $\zeta_{2}=\rho_{2} \mathrm{e}^{\mathrm{i} \theta_{2}}$. The angles $\theta_{1}$ and $\theta_{2}$ are the polar angles in the $\zeta_{1^{-}}$and $\zeta_{2}$-planes, respectively. $\rho_{1}=\rho_{2}=1$ on the hole boundary.

Assuming that $\zeta_{1}=\zeta_{2}=\zeta=\sigma=\mathrm{e}^{\mathrm{i} \theta}$ along the unit circle, the relationships between $\zeta, \zeta_{1}$ and $\zeta_{2}$ can also be established

$$
\begin{aligned}
& \gamma_{1} R\left(\zeta_{1}+\sum_{k=1}^{n} C_{k} \zeta_{1}^{-k}\right)+\delta_{1} \bar{R}\left(\frac{1}{\zeta_{1}}+\sum_{k=1}^{n} \bar{C}_{k} \zeta_{1}^{k}\right)=\gamma_{1} R\left(\zeta+\sum_{k=1}^{n} C_{k} \zeta^{-k}\right)+\delta_{1} \bar{R}\left(\bar{\zeta}+\sum_{k=1}^{n} \bar{C}_{k} \bar{\zeta}^{-k}\right) \\
& \gamma_{2} R\left(\zeta_{2}+\sum_{k=1}^{n} C_{k} \zeta_{2}^{-k}\right)+\delta_{2} \bar{R}\left(\frac{1}{\zeta_{2}}+\sum_{k=1}^{n} \bar{C}_{k} \zeta_{2}^{k}\right)=\gamma_{2} R\left(\zeta+\sum_{k=1}^{n} C_{k} \zeta^{-k}\right)+\delta_{2} \bar{R}\left(\bar{\zeta}+\sum_{k=1}^{n} \bar{C}_{k} \bar{\zeta}^{-k}\right)
\end{aligned}
$$

Equations (2.8) are suitable for any point in the region $|\zeta| \geqslant 1$. Given a point $\zeta$, then points $\zeta_{1}$ and $\zeta_{2}$ can be determined by Eqs. (2.8). $z, z_{1}$, and $z_{2}$ can be calculated by Eqs. (2.5) and (2.7), respectively.

\subsection{Calculation of the analytical solution of stress}

For the plane stress problem of an orthotropic plane, when the body forces are not taken into consideration, the compatibility equation for Airy's stress function $F=F\left(x^{\prime}, y^{\prime}\right)$ in the local coordinates $x^{\prime} o y^{\prime}$ can be given as (Lekhnitskii, 1968, 1981)

$$
a_{22}^{\prime} \frac{\partial^{4} F}{\partial x^{\prime 4}}-2 a_{26}^{\prime} \frac{\partial^{4} F}{\partial x^{\prime 3} \partial y^{\prime}}+\left(2 a_{12}^{\prime}+a_{66}^{\prime}\right) \frac{\partial^{4} F}{\partial x^{\prime 2} \partial y^{\prime 2}}-2 a_{16}^{\prime} \frac{\partial^{4} F}{\partial x^{\prime} \partial y^{\prime 3}}+a_{11}^{\prime} \frac{\partial^{4} F}{\partial y^{\prime 4}}=0
$$

where $a_{i j}^{\prime}$ are constants related to the material properties. The solution to Eq. (2.9) is related to the roots of the following characteristic equation

$$
a_{11}^{\prime} \mu^{\prime 4}-2 a_{16}^{\prime} \mu^{\prime 3}+\left(2 a_{12}^{\prime}+a_{66}^{\prime}\right) \mu^{\prime 2}-2 a_{26}^{\prime} \mu^{\prime}+a_{22}^{\prime}=0
$$

The four conjugate complex roots in Eq. (2.10) are $\mu_{1}^{\prime}, \bar{\mu}_{1}^{\prime}, \mu_{2}^{\prime}$ and ${\overline{\mu^{\prime}}}_{2}$, which can be calculated by the principal elastic constants. However, the complex roots in the global coordinates xoy should be calculated due to the change of the fiber orientation. When the local coordinates $x^{\prime} o y^{\prime}$ overlap the global coordinates xoy after rotating $\varphi$ degrees counter clockwise, $\mu_{1}$ and $\mu_{2}$ in the global coordinates can be given as (Lekhnitskii, 1968)

$$
\mu_{1}=\frac{\mu_{1}^{\prime} \cos \varphi-\sin \varphi}{\cos \varphi+\mu_{1}^{\prime} \sin \varphi} \quad \mu_{2}=\frac{\mu_{2}^{\prime} \cos \varphi-\sin \varphi}{\cos \varphi+\mu_{2}^{\prime} \sin \varphi}
$$


In this paper, only the situation of $\mu_{1} \neq \mu_{2}$ is discussed. The solution to Eq. (2.9) can be expressed by the two analytical functions $F_{1}\left(z_{1}\right)$ and $F_{2}\left(z_{2}\right)$ as

$$
F=2 \operatorname{Re}\left[F_{1}\left(z_{1}\right)+F_{2}\left(z_{2}\right)\right]
$$

where $z_{1}$ and $z_{2}$ are exactly the same as given in the previous Sections.

Let $\Phi_{1}\left(z_{1}\right)=d F_{1}\left(z_{1}\right) / d z_{1}$ and $\Phi_{2}\left(z_{2}\right)=d F_{2}\left(z_{2}\right) / d z_{2}$, respectively. The stress boundary conditions on the edge of the hole expressed by $\Phi_{1}\left(z_{1}\right)$ and $\Phi_{2}\left(z_{2}\right)$ can be given as

$$
\begin{aligned}
& 2 \operatorname{Re}\left[\Phi_{1}\left(z_{1}\right)+\Phi_{2}\left(z_{2}\right)\right]=f_{1} \\
& 2 \operatorname{Re}\left[\mu_{1} \Phi_{1}\left(z_{1}\right)+\mu_{2} \Phi_{2}\left(z_{2}\right)\right]=f_{2}
\end{aligned}
$$

where $z_{1}$ and $z_{2}$ are two points on the boundary.

The problem discussed here is an infinite field with holes, and no loads exist along the edge of the hole. Therefore, $f_{1}=f_{2}=0$, and $\Phi_{1}\left(z_{1}\right)$ and $\Phi_{2}\left(z_{2}\right)$ can be given in the following form

$$
\Phi_{1}\left(z_{1}\right)=B^{*} z_{1}+\Phi_{1}^{0}\left(z_{1}\right) \quad \Phi_{2}\left(z_{2}\right)=\left(B^{\prime *}+\mathrm{i} C^{\prime *}\right) z_{2}+\Phi_{2}^{0}\left(z_{2}\right)
$$

where $B^{*}, B^{\prime *}$, and $C^{\prime *}$ can be determined according to the stress components acting at infinity (i.e., $\sigma_{x}^{\infty}, \sigma_{y}^{\infty}$ and $\tau_{x y}^{\infty}$ ) as

$$
\begin{aligned}
B^{*} & =\frac{\sigma_{x}^{\infty}+\left(\alpha_{2}^{2}+\beta_{2}^{2}\right) \sigma_{y}^{\infty}+2 \alpha_{2} \tau_{x y}^{\infty}}{2\left[\left(\alpha_{2}-\alpha_{1}\right)^{2}+\left(\beta_{2}^{2}-\beta_{1}^{2}\right)\right]} \\
B^{\prime *} & =\frac{\left(\alpha_{1}^{2}-\beta_{1}^{2}-2 \alpha_{1} \alpha_{2}\right) \sigma_{y}^{\infty}-\sigma_{x}^{\infty}-2 \alpha_{2} \tau_{x y}^{\infty}}{2\left[\left(\alpha_{2}-\alpha_{1}\right)^{2}+\left(\beta_{2}^{2}-\beta_{1}^{2}\right)\right]} \\
C^{*} & =\frac{\left(\alpha_{1}-\alpha_{2}\right) \sigma_{x}^{\infty}+\left[\alpha_{2}\left(\alpha_{1}^{2}-\beta_{1}^{2}\right)-\alpha_{1}\left(\alpha_{2}^{2}-\beta_{2}^{2}\right)\right] \sigma_{y}^{\infty}+\left[\left(\alpha_{1}^{2}-\beta_{1}^{2}\right)-\left(\alpha_{2}^{2}-\beta_{2}^{2}\right)\right] \tau_{x y}^{\infty}}{2 \beta_{2}\left[\left(\alpha_{2}-\alpha_{1}\right)^{2}+\left(\beta_{2}^{2}-\beta_{1}^{2}\right)\right]}
\end{aligned}
$$

Here, $\Phi_{1}^{0}\left(z_{1}\right)$ and $\Phi_{2}^{0}\left(z_{2}\right)$ in Eqs. (2.14) should have the following form after substituting $z_{1}=\omega_{1}\left(\zeta_{1}\right)$ and $z_{2}=\omega_{2}\left(\zeta_{2}\right)$ into them

$$
\Phi_{1}^{0}\left(z_{1}\right)=\Phi_{1}^{0}\left[\omega_{1}\left(\zeta_{1}\right)\right]=\sum_{k=0}^{\infty} a_{k} \zeta_{1}^{-k} \quad \Phi_{2}^{0}\left(z_{2}\right)=\Phi_{2}^{0}\left[\omega_{2}\left(\zeta_{2}\right)\right]=\sum_{k=0}^{\infty} b_{k} \zeta_{2}^{-k}
$$

where $a_{k}=a_{1 k}+\mathrm{i} a_{2 k}, b_{k}=b_{1 k}+\mathrm{i} b_{2 k}, a_{1 k}, a_{2 k}, b_{1 k}$ and $b_{2 k}$ are undetermined real constants $(k=0, \ldots, \infty)$.

Along the edge of the hole, $\zeta_{1}=\sigma=\mathrm{e}^{\mathrm{i} \theta}$ and $\zeta_{2}=\sigma=\mathrm{e}^{\mathrm{i} \theta}$. The undetermined coefficients $a_{k}$ and $b_{k}$ can be determined from Eqs. (2.13)-(2.15) by using the power-series method. Then, the stress components $\sigma_{x}, \sigma_{y}$ and $\tau_{x y}$ in the Cartesian coordinates can be determined by the following equations ( $\mathrm{Lu}$ et al., 2015)

$$
\begin{array}{ll}
\sigma_{x}=2 \operatorname{Re}\left[\mu_{1}^{2} \Phi_{1}^{\prime}\left(z_{1}\right)+\mu_{2}^{2} \Phi_{2}^{\prime}\left(z_{2}\right)\right] & \sigma_{y}=2 \operatorname{Re}\left[\Phi_{1}^{\prime}\left(z_{1}\right)+\Phi_{2}^{\prime}\left(z_{2}\right)\right] \\
\tau_{x y}=-2 \operatorname{Re}\left[\mu_{1} \Phi_{1}^{\prime}\left(z_{1}\right)+\mu_{2} \Phi_{2}^{\prime}\left(z_{2}\right)\right] &
\end{array}
$$

where

$$
\begin{aligned}
& \Phi_{1}^{\prime}\left(z_{1}\right)=B^{*}-\sum_{k=1}^{n} k a_{k} \zeta_{1}^{-k-1} /\left[\gamma_{1} R\left(1-\sum_{k=1}^{n} k C_{k} \zeta_{1}^{-k-1}\right)+\delta_{1} \bar{R}\left(-\frac{1}{\zeta_{1}^{2}}+\sum_{k=1}^{n} k \bar{C}_{k} \zeta_{1}^{k-1}\right)\right] \\
& \Phi_{2}^{\prime}\left(z_{2}\right)=B^{\prime *}+\mathrm{i} C^{\prime *}-\sum_{k=1}^{n} k b_{k} \zeta_{2}^{-k-1} /\left[\gamma_{2} R\left(1-\sum_{k=1}^{n} k C_{k} \zeta_{2}^{-k-1}\right)+\delta_{2} \bar{R}\left(-\frac{1}{\zeta_{2}^{2}}+\sum_{k=1}^{n} k \bar{C}_{k} \zeta_{2}^{k-1}\right)\right]
\end{aligned}
$$


The stress components $\sigma_{\rho}, \sigma_{\theta}$ and $\tau_{\rho \theta}$ in orthogonal curvilinear coordinates can be determined by the following equations

$$
\sigma_{\rho}+\sigma_{\theta}=\sigma_{x}+\sigma_{y} \quad \sigma_{\theta}-\sigma_{\rho}+2 \mathrm{i} \tau_{\rho \theta}=\frac{\zeta^{2}}{\rho^{2}} \frac{\omega^{\prime}(\zeta)}{\omega^{\prime}(\zeta)}\left(\sigma_{y}-\sigma_{x}+2 \mathrm{i} \tau_{x y}\right)
$$

Along the hole boundary where $\zeta_{1}=\zeta_{2}=\zeta=\sigma=\mathrm{e}^{\mathrm{i} \theta}$ and $\rho=1$, the analytical solution of stress can be solved easily by Eqs. (2.16)-(2.18). In this way, when the mapping functions are known and the material parameters and external loads are given, no matter how the fiber orientation angle and hole orientation angle change, the analytical solution of the stress along and near the hole boundary can be calculated. In addition, this analytical solution of the stress is suitable for an arbitrarily shaped hole.

\subsection{Optimization}

Tangential stress concentration along the boundary of the hole will cause damage if its magnitude exceeds the material strength. Consequently, the optimization criterion we take is that the absolute maximum value of the tangential stress along the hole boundary should be kept at the minimum. In the optimization, the fiber orientation angle $\varphi$ and the hole orientation angle $\alpha$ are the unknown variables. A set of the initial values of $\varphi$ and $\alpha$ are given; then, the tangential stress with the maximum absolute value $\max \left|\sigma_{\theta}\right|$ could be obtained through the complex variable method. By revising the values of design variables $\varphi$ and $\alpha$, the final values of $\hat{\varphi}$ and $\widehat{\alpha}$ that lead to the minimal value of $\max \left|\sigma_{\theta}\right|$ should be the optimized results. The mathematical model could be expressed as follows

$$
\begin{aligned}
& F(X)=\max _{\theta \in[0,2 \pi]}\left|\sigma_{\theta}\right| \\
& \min F(X) \quad X \in D=\{\varphi, \alpha\} \\
& \text { s.t. } \quad 0 \leqslant \varphi \leqslant 2 \pi \quad 0 \leqslant \alpha \leqslant 2 \pi
\end{aligned}
$$

The original DE algorithm (Storn and Price, 1997) is used in the computations. It can be written as DE/rand/1/bin. As defined by Storn and Price, the DE algorithm is characterized by three main parameters $N P, F$ and $C R$ as

$$
\begin{aligned}
& x_{i, G} i=1,2, \ldots, N P \\
& v_{i, G+1}=x_{r 1, G}+F\left(x_{r 2, G}-x_{r 3, G}\right) \\
& u_{j i, G+1}=\left\{\begin{array}{llll}
v_{j i, G+1} & \text { if } \quad(\operatorname{randb}(j) \leqslant C R) & \vee & j=\operatorname{rnbr}(i) \\
x_{j i, G} & \text { if } \quad(\operatorname{randb}(j)>C R) & \wedge & j \neq \operatorname{rnbr}(i)
\end{array}\right.
\end{aligned}
$$

In this paper, the number of population vectors $N P$ equals 200 . The real and constant factor $F$ equals 0.8 , and the crossover constant $C R$ is equal to 0.5 .

\section{Examples}

As shown in Fig. 1, the external loads are chosen as $\sigma_{x}^{\infty}: \sigma_{y}^{\infty}=2: 7$ and $\tau_{x y}^{\infty}=0$. Sign convention is defined as positive for tension and negative for compression. It can be seen from the results of the following examples that all of the maximum tangential stresses are tensile stresses. The values of the independent principal elastic constants in the local coordinates $x^{\prime} o y^{\prime}$ are: $E_{1}=1.4 \cdot 10^{5} \mathrm{~Pa}, v_{12}=0.46$ and $G_{12}=1.2 \cdot 10^{4} \mathrm{~Pa}$, where the subscripts 1 and 2 represent the directions along the $x^{\prime}$ - and $y^{\prime}$-axis, respectively. To analyze the effect of Young's modulus on the optimization results, we take different values of $E_{2} / E_{1}$, i.e., $0.2,0.5,2.0$ and 5.0. The 
material coefficients in Eq. (2.9) can be obtained by the three elastic constants as $a_{11}^{\prime}=1 / E_{1}$, $a_{22}^{\prime}=1 / E_{2}, a_{16}^{\prime}=a_{26}^{\prime}=0, a_{12}^{\prime}=-v_{12} / E_{1}$, and $a_{66}^{\prime}=1 / G_{12}$ (Lekhnitskii, 1968; Chen, 1994).

The hexagonal hole and the elliptical hole are selected as examples. The size of the hole has no influence on the calculation of the stress field, because the domain occupied by the plate is infinite. In order not to lose the generality, take $R_{1}=1.0$. The mapping function of Eq. (2.5) can be described as

$$
z=\omega(\zeta)=1.0 \mathrm{e}^{\mathrm{i} \alpha}\left(\zeta+\sum_{k=1}^{n} C_{k} \zeta^{-k}\right)
$$

The imaginary part of the coefficient $C_{k}$ is equal to zero, because the hole shapes of the examples are all symmetric about the $x_{1}$-axis.

For the elliptical hole: $a / b=1.5, n=1, C_{1}=(a-b) /(a+b)=0.2$.

For the hexagonal hole: $n=29, C_{5}=0.0667, C_{11}=0.0101, C_{17}=0.0036, C_{23}=0.0018$ and $C_{29}=0.0010$ (Savin, 1961). The other $C_{k}$ are all equal to zero.

\subsection{Optimization of the fiber orientation angle $\varphi$}

In this Section, only the fiber orientation angle $\varphi$ is taken as the design variable, while the external loads and hole orientation angle $\alpha$ are given. The hole orientation angle $\alpha$ shown in Fig. 1 is set to $0^{\circ}$ (see Fig. 2), meaning that the coordinates $x_{1} o y_{1}$ coincide with $x o y$. The local coordinates $x^{\prime} y^{\prime}$ still follow the fiber orientation, coinciding with $x o y$ after rotation by $\varphi$ degrees counterclockwise. The scope of the optimized variable is $\varphi \in 0^{\circ}-90^{\circ}$ because of the symmetry of the external loads and the hole shape.
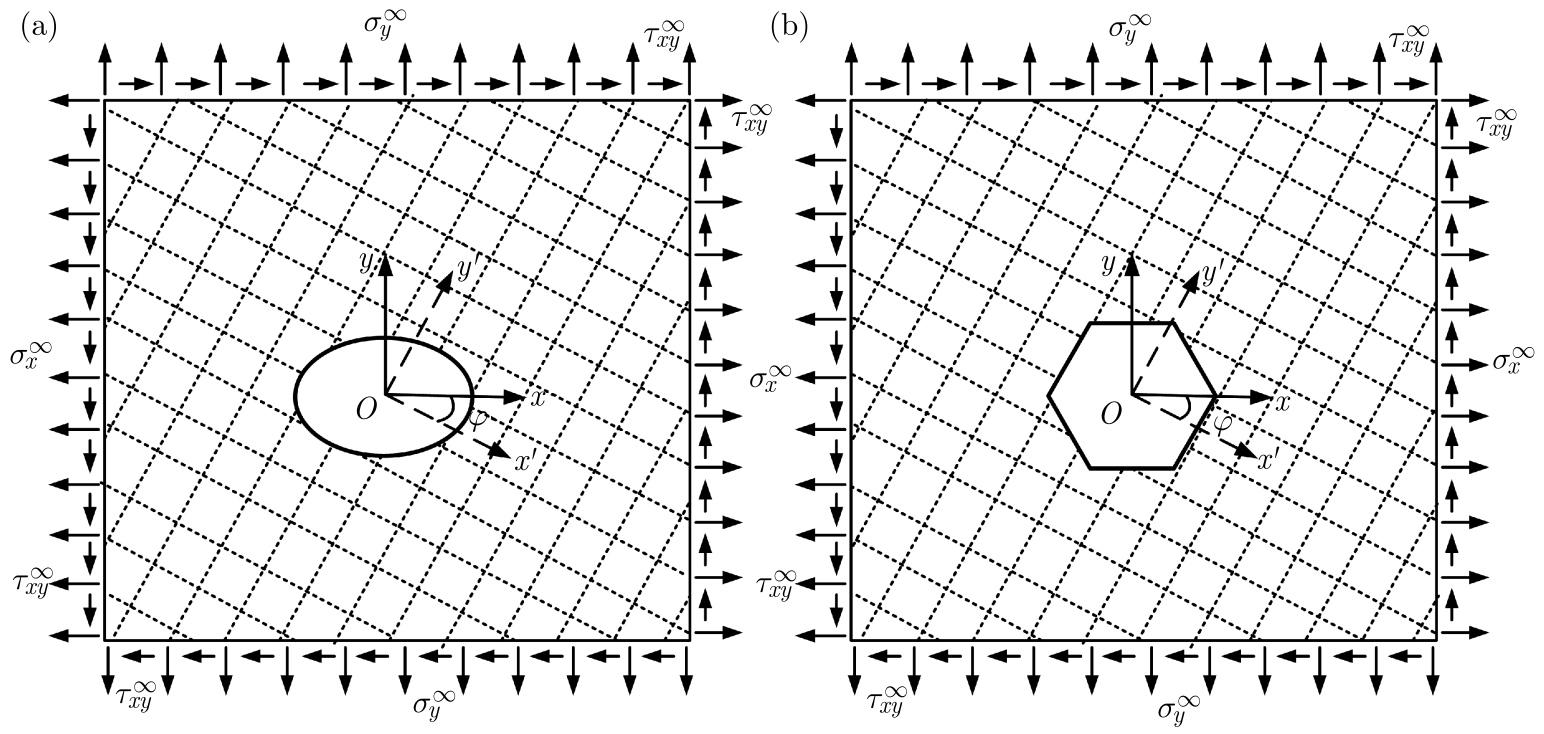

Fig. 2. The location of holes with two coordinates: (a) elliptical hole, (b) hexagonal hole

\subsubsection{Elliptical hole}

The optimal fiber orientation and its corresponding tangential stress along the boundary of the elliptical hole are illustrated in Figs. 3 and 4, respectively. The optimized fiber orientation angles and the maximum tangential stresses are listed in Table 1. The global coordinates xoy in Fig. 3 are in accordance with that in Fig. 2a, and the oblique lines in Fig. 3 represent the fiber directions in the $x^{\prime}$-axis (Fig. 2a) for different values of $E_{2} / E_{1}$. It should be noted that the 


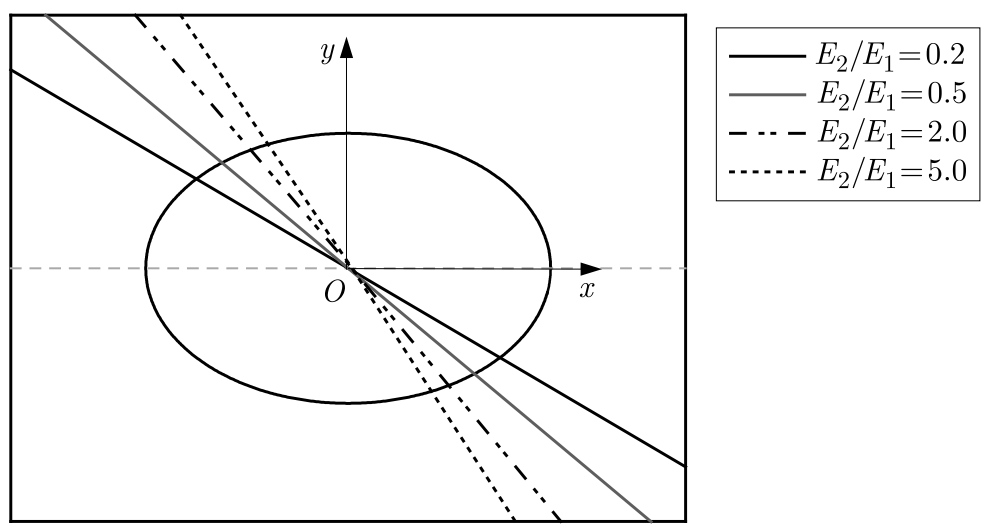

Fig. 3. The optimal fiber orientation of the orthotropic plate with the elliptical hole

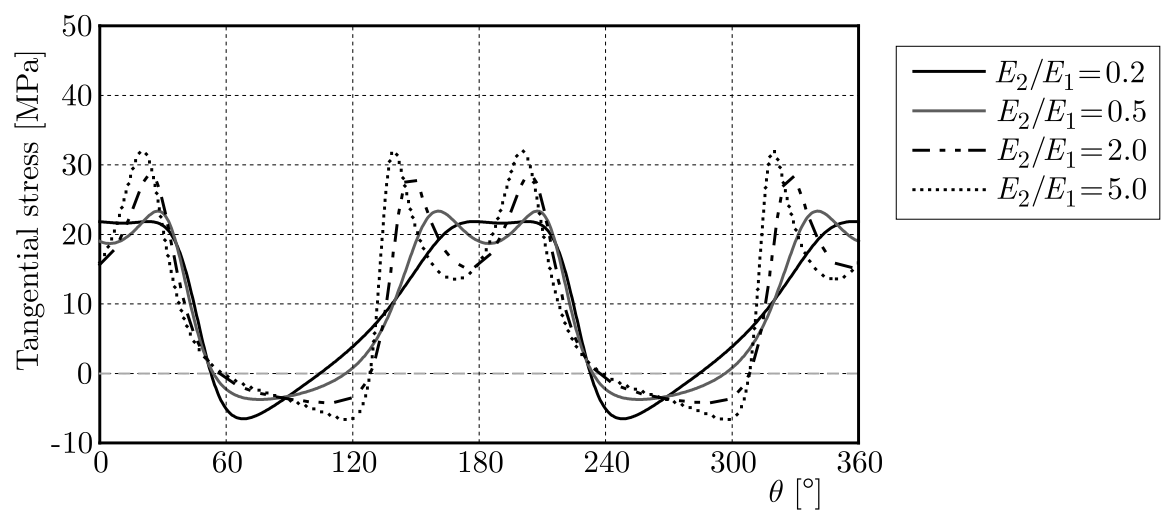

Fig. 4. Tangential stresses along the boundary of the elliptical hole at the optimal fiber orientation

Table 1. The optimal fiber orientation angles and the $\max \left|\sigma_{\theta}\right|$ of an orthotropic plate with the elliptical hole

\begin{tabular}{|l|c|c|c|c|}
\hline \multirow{2}{*}{} & \multicolumn{4}{|c|}{$E_{2} / E_{1}$} \\
\cline { 2 - 5 } & 0.2 & 0.5 & 2.0 & 5.0 \\
\hline \hline$\varphi\left[^{\circ}\right]$ & 30.46 & 39.89 & 49.96 & 56.49 \\
\hline $\max \left|\sigma_{\theta}\right|[\mathrm{MPa}]$ & 21.84 & 23.34 & 28.50 & 32.04 \\
\hline
\end{tabular}

angle $\theta$ in Fig. 4 is the polar angle in the $\zeta$-plane and $\theta=0^{\circ}$ corresponds to the intersection of the positive $x$-axis and the excavation boundary.

It can be seen from Figs. 3 and 4 and Table 1 that both the optimal fiber orientation angle $\varphi$ and the maximum tangential stress increase with the increasing value of $E_{2} / E_{1}$. The maximum tangential stress concentration is evident in approximately $30^{\circ}, 150^{\circ}, 210^{\circ}$ and $330^{\circ}$, not similar to the isotropic plate, where the maximum tangential stress usually occurs at $0^{\circ}$ and $180^{\circ}$. Under the given loading condition, the compressive stress can be found around the intersection of the $y$-axis and the hole boundary with a smaller magnitude than the tensile stress.

Considering that there is only one variable in this case, the optimal fiber orientation angle $\varphi$ can be obtained without any optimization algorithm. Figure 5 illustrates the maximum absolute tangential stress $\max \left|\sigma_{\theta}\right|$ for different fiber orientation angles, showing that the minimum value of $\max \left|\sigma_{\theta}\right|$ and the corresponding fiber orientation angles are identical with the results listed in Table 1, which verifies the results obtained by the DE algorithm.

For an elliptical hole, the stresses have also been analyzed by Ukadgaonker and Rao (2000). To verify the fundamental theories of Section 2, we compared the normalized stresses of $\sigma_{x}$, $\sigma_{y}$ and $\tau_{x y}$ along the hole boundary with the results obtained by Ukadgaonker and Rao (2000, 


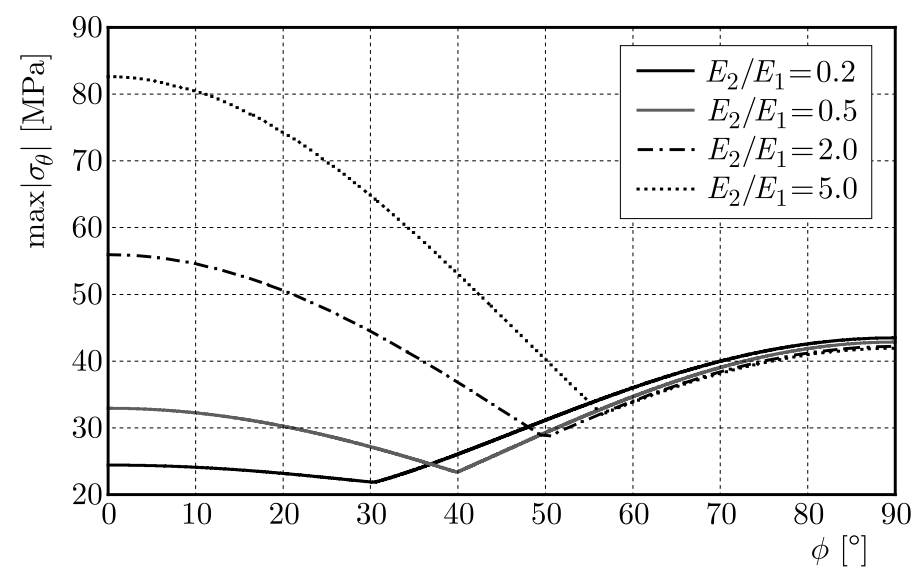

Fig. 5. The $\max \left|\sigma_{\theta}\right|$ for different fiber orientation angles

Table 2. Normalized stresses of $\sigma_{x}, \sigma_{y}$ and $\tau_{x y}$ for equi-biaxial tension on a graphite/epoxy plate, $60^{\circ}$ fibers containing an elliptical hole $(a / b=2)$

\begin{tabular}{|c|c|c|c|c|c|c|}
\hline \multirow{2}{*}{$\theta[\mathrm{deg}]$} & \multicolumn{3}{|c|}{ Ukadgaonker and Rao $(2000)$} & \multicolumn{3}{|c|}{ Zhang et al. } \\
\cline { 2 - 7 } & $\sigma_{x}$ & $\sigma_{y}$ & $\tau_{x y}$ & $\sigma_{x}$ & $\sigma_{y}$ & $\tau_{x y}$ \\
\hline \hline 0 & 0 & 3.44 & 0 & 0 & 3.4393 & 0 \\
\hline 20 & 0.82 & 1.54 & -1.12 & 0.8172 & 1.5421 & -1.1226 \\
\hline 40 & 1.46 & 0.52 & -0.87 & 1.4589 & 0.518 & -0.8693 \\
\hline 60 & 1.54 & 0.13 & -0.45 & 1.5423 & 0.1285 & -0.4452 \\
\hline 80 & 1.41 & 0.01 & -0.12 & 1.4076 & 0.0109 & -0.1241 \\
\hline 90 & 1.3 & 0 & 0 & 1.3033 & 0 & 0 \\
\hline 100 & 1.18 & 0.01 & 0.1 & 1.1812 & 0.0092 & 0.1041 \\
\hline 120 & 0.89 & 0.07 & 0.26 & 0.8867 & 0.0739 & 0.256 \\
\hline 140 & 0.57 & 0.2 & 0.34 & 0.5651 & 0.2007 & 0.3367 \\
\hline 160 & 1.23 & 2.32 & 1.69 & 1.2272 & 2.316 & 1.6859 \\
\hline 180 & 0 & 3.44 & 0 & 0 & 3.4393 & 0 \\
\hline
\end{tabular}

page 348, Table 3) in Table 2. Taking the same values of material parameters and external loadings, Table 2 shows that the results obtained by the two papers are in a very close agreement.

\subsubsection{Hexagonal hole}

The optimized fiber orientation angles and their corresponding tangential stresses along the boundary of the hexagonal hole are illustrated in Figs. 6 and 7, respectively. The maximum tangential stress occurs at the points marked with an asterisk. When the ratios of the elastic moduli are $E_{2} / E_{1}=0.2,0.5,2.0$ and 5.0, the corresponding optimal fiber orientation angles are $\varphi=32.15^{\circ}, 40.47^{\circ}, 42.74^{\circ}$ and $57.90^{\circ}$, respectively (shown in Fig. 6), which shows a great difference for the different ratios of $E_{2} / E_{1}$. Figure 7 illustrates that the tangential stress reaches the corresponding extreme values in/near the corner points. The maximum tangential stresses for different $E_{2} / E_{1}$ are found in different positions but are still around the corner points, and the values of the maximum tangential stress range from $42.89 \mathrm{MPa}\left(E_{2} / E_{1}=0.5\right)$ to $70.77 \mathrm{MPa}$ $\left(E_{2} / E_{1}=5.0\right)$, meaning that the location and magnitude of the maximum tangential stress are highly dependent on Young's modulus for a given hole shape and loading condition. We can also see that the tangential stresses along the boundary are mainly tensile stresses with the exception of the two sides $\mathrm{BC}$ and $\mathrm{EF}$, which are parallel to the direction of the minimum loading. Moreover, the magnitude of the compressive stress is much smaller than that of the tensile stress. 
Comparing with the results in Fig. 4, the stress concentration along the boundary of the hexagonal hole is much larger than that along the boundary of the elliptical hole because there are sharp corners on the hexagonal hole.
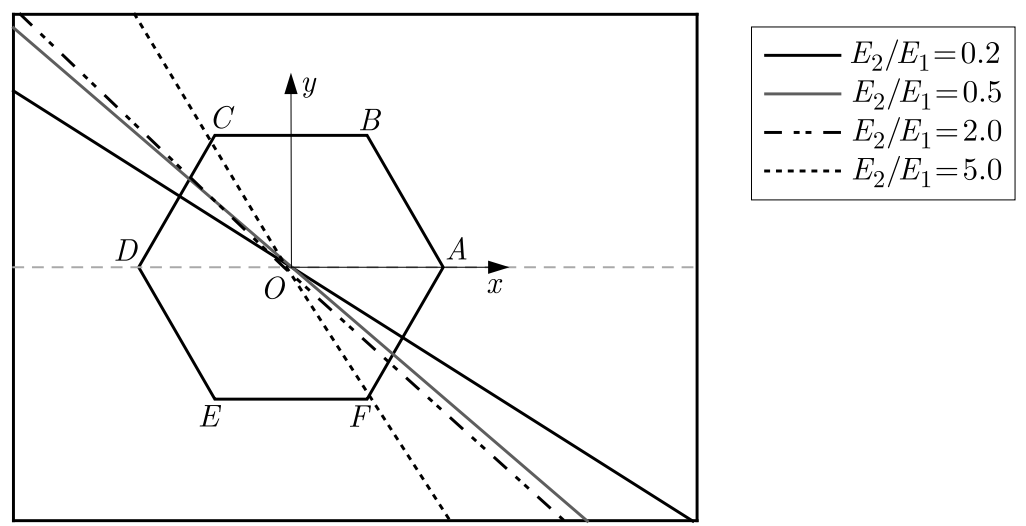

Fig. 6. The optimal fiber orientation of the orthotropic plate with the hexagonal hole
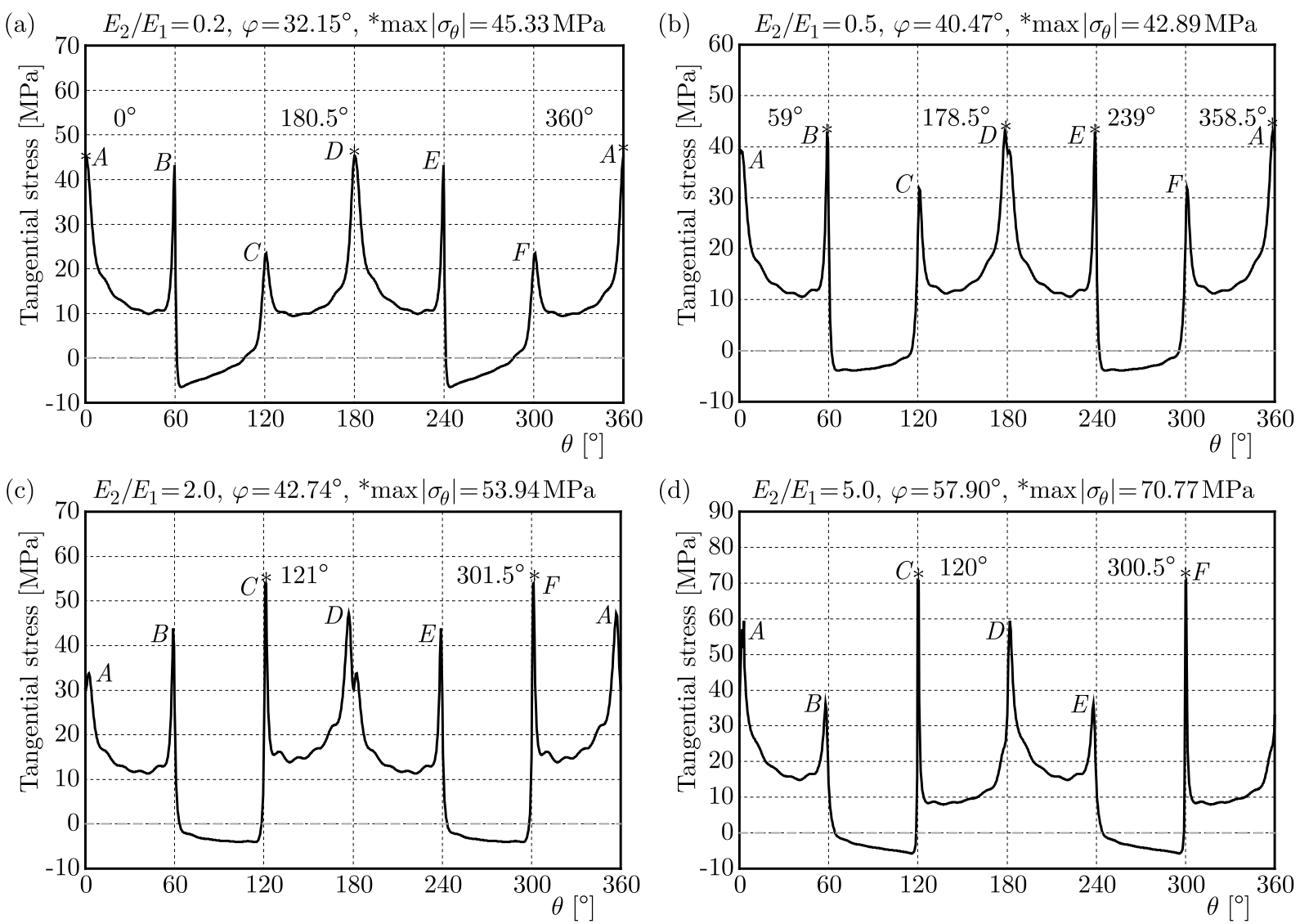

Fig. 7. Tangential stresses along the boundary of the hexagonal hole at the optimal fiber orientation

\subsection{Optimization of the fiber and hole orientation angles}

Considering that the hole orientation angle also influences the tangential stress along the boundary of the hole, both the fiber orientation angle and the hole orientation angle are chosen as the design variables in this Section with the external loads given (refer Fig. 1). 


\subsubsection{Elliptical hole}

Because of the symmetry of the external loads and the elliptical hole, the scopes of optimized variables are given as $\alpha \in 0^{\circ}-180^{\circ}$ and $\varphi \in 0^{\circ}-90^{\circ}$. The optimal fiber and hole orientation angles and the corresponding tangential stress along the boundary of the hole are illustrated in Figs. 8 and 9, and Table 3, respectively.

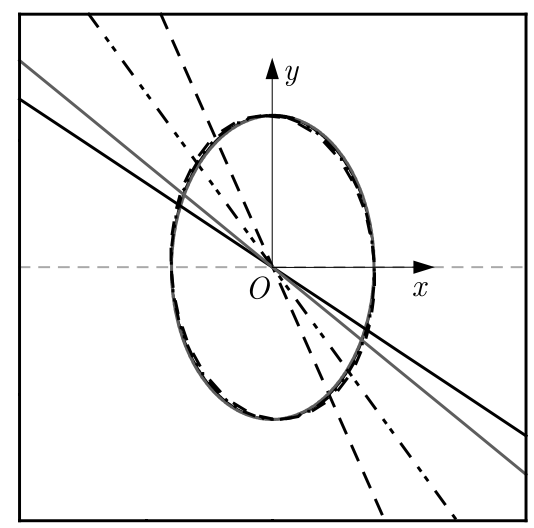

$$
\begin{array}{r}
-E_{2} / E_{1}=0.2 \\
-E_{2} / E_{1}=0.5 \\
---E_{2} / E_{1}=2.0 \\
---E_{2} / E_{1}=5.0
\end{array}
$$

Fig. 8. The optimal fiber and hole orientation angles of the orthotropic plate with the elliptical hole

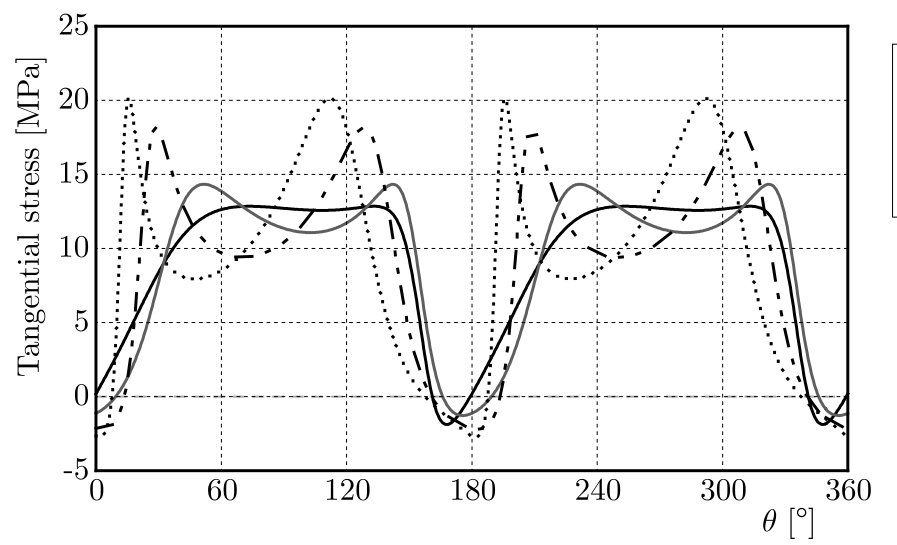

$$
\begin{aligned}
&-E_{2} / E_{1}=0.2 \\
&-E_{2} / E_{1}=0.5 \\
&-\cdots-E_{2} / E_{1}=2.0 \\
& \cdots \cdots \cdots \cdot E_{2} / E_{1}=5.0
\end{aligned}
$$

Fig. 9. Tangential stresses along the boundary of the elliptical hole at the optimal fiber and hole orientation

Table 3. The optimal fiber and hole orientation and the $\max \left|\sigma_{\theta}\right|$ of the orthotropic plate with the elliptical hole

\begin{tabular}{|l|c|c|c|c|}
\hline \multirow{2}{*}{} & \multicolumn{4}{|c|}{$E_{2} / E_{1}$} \\
\cline { 2 - 5 } & 0.2 & 0.5 & 2.0 & 5.0 \\
\hline \hline$\varphi\left[^{\circ}\right]$ & 33.64 & 39.25 & 53.98 & 66.13 \\
\hline$\alpha\left[^{\circ}\right]$ & 90.95 & 89.29 & 92.43 & 94.84 \\
\hline $\max \left|\sigma_{\theta}\right|[\mathrm{MPa}]$ & 12.85 & 14.33 & 18.12 & 20.11 \\
\hline
\end{tabular}

It can be seen from Fig. 8 and Table 3 that the optimal fiber orientation angle increases greatly with an increase in the ratio of $E_{1} / E_{2}$. Nevertheless, the hole orientation angles are all near the same degree of $90^{\circ}$, that is to say, the major axis of the elliptical hole should be set in the direction of the maximum external load. It can also be verified by comparing Figs. 4 and 9 . Under the given loadings, vertical elliptical holes (Fig. 9) always produce smaller tangential stress concentration compared to the horizontal ones (Fig. 4). In addition, the similar laws of the two cases are that the maximum tangential stress increases with the ratio of $E_{2} / E_{1}$ and compressive stress is found in Fig. 9 as well. 


\subsubsection{Hexagonal hole}

Because a hexagonal hole has more symmetry axes, the scopes of the optimized variables are reduced to $\alpha \in 0^{\circ}-60^{\circ}$ and $\varphi \in 0^{\circ}-90^{\circ}$. Figures 10-13 show the optimal placements of the fiber and hole orientation angles and the corresponding tangential stress along the hole boundary. It should be noted that the angle $\theta=0^{\circ}$ corresponds to the intersection of the positive $x_{1}$-axis and the hole in this case.

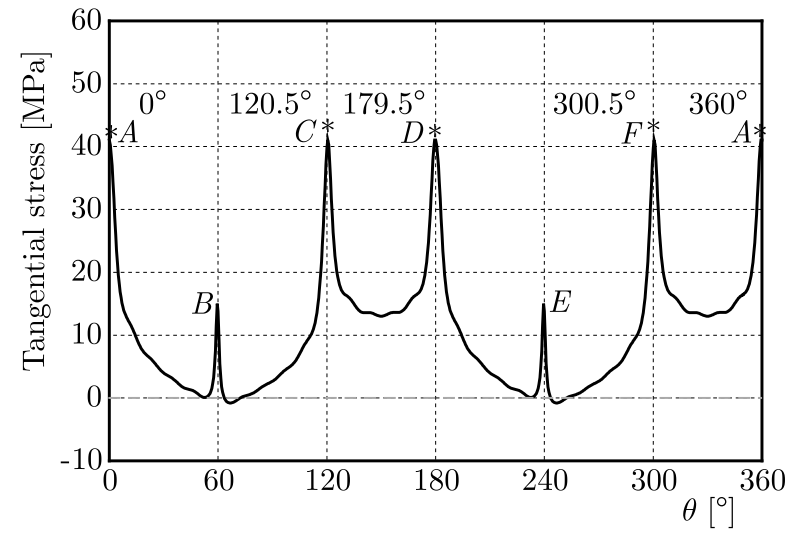

$E_{2} / E_{1}=0.2, \varphi=8.22^{\circ}, \alpha=27.97^{\circ},{ }^{*} \max \left|\sigma_{\theta}\right|=41.02 \mathrm{MPa}$

Fig. 10. The optimal placement of fiber and hole orientation angles of orthotropic plates and the tangential stresses along the boundary of the hexagonal hole when $E_{2} / E_{1}=0.2$

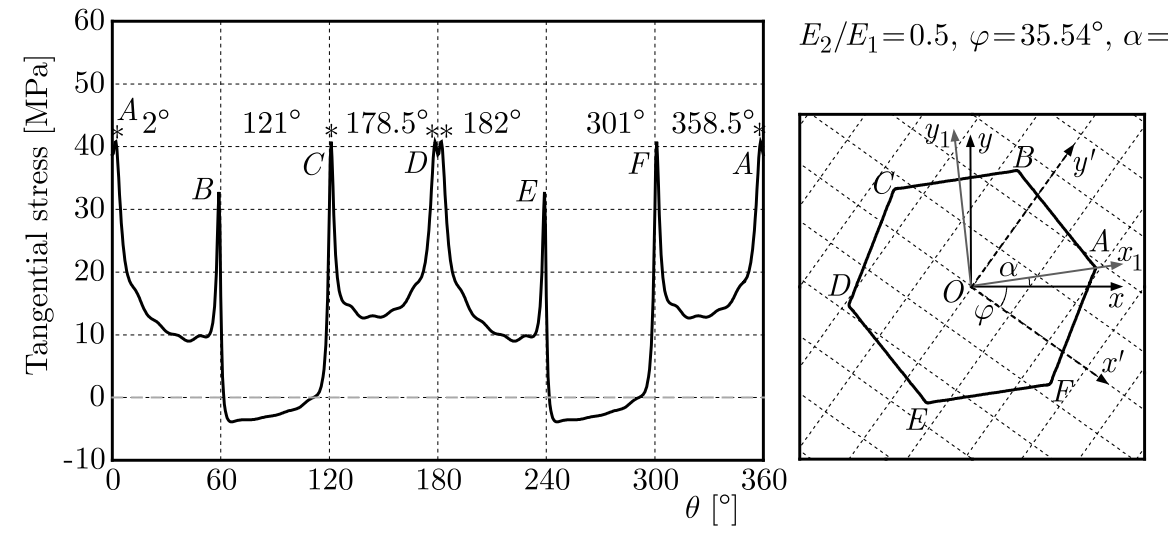

Fig. 11. The optimal placement of fiber and hole orientation angles of orthotropic plates and the tangential stresses along the boundary of the hexagonal hole when $E_{2} / E_{1}=0.5$

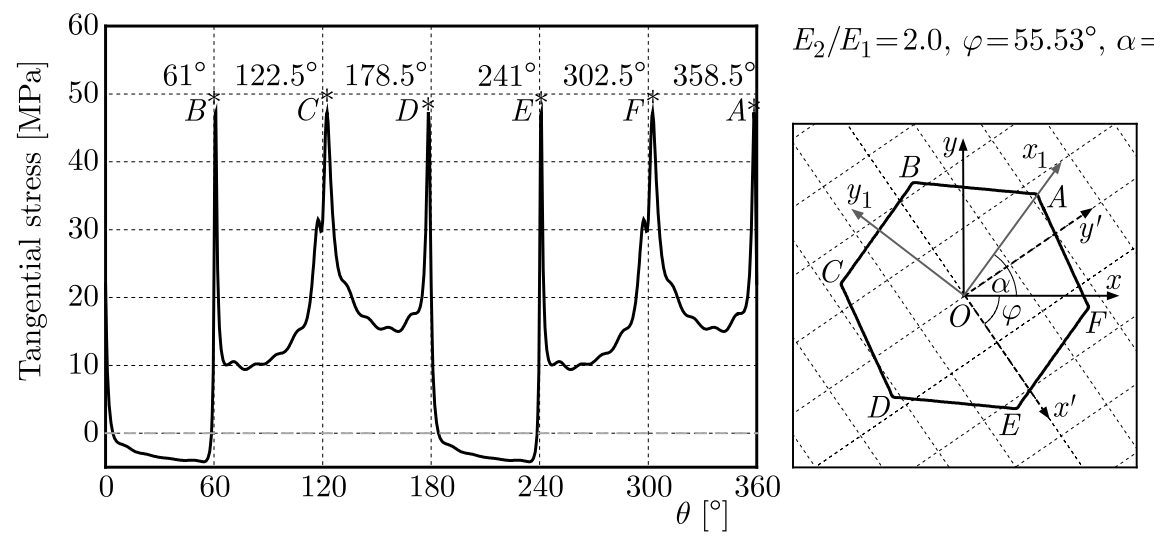

Fig. 12. The optimal placement of fiber and hole orientation angles of orthotropic plates and the tangential stresses along the boundary of the hexagonal hole when $E_{2} / E_{1}=2.0$

It can be seen from Figs. 10-13 that both the optimized fiber orientation angle and the hole orientation angle are apparently different for different ratios of $E_{2} / E_{1}$. Under the given inequi- 


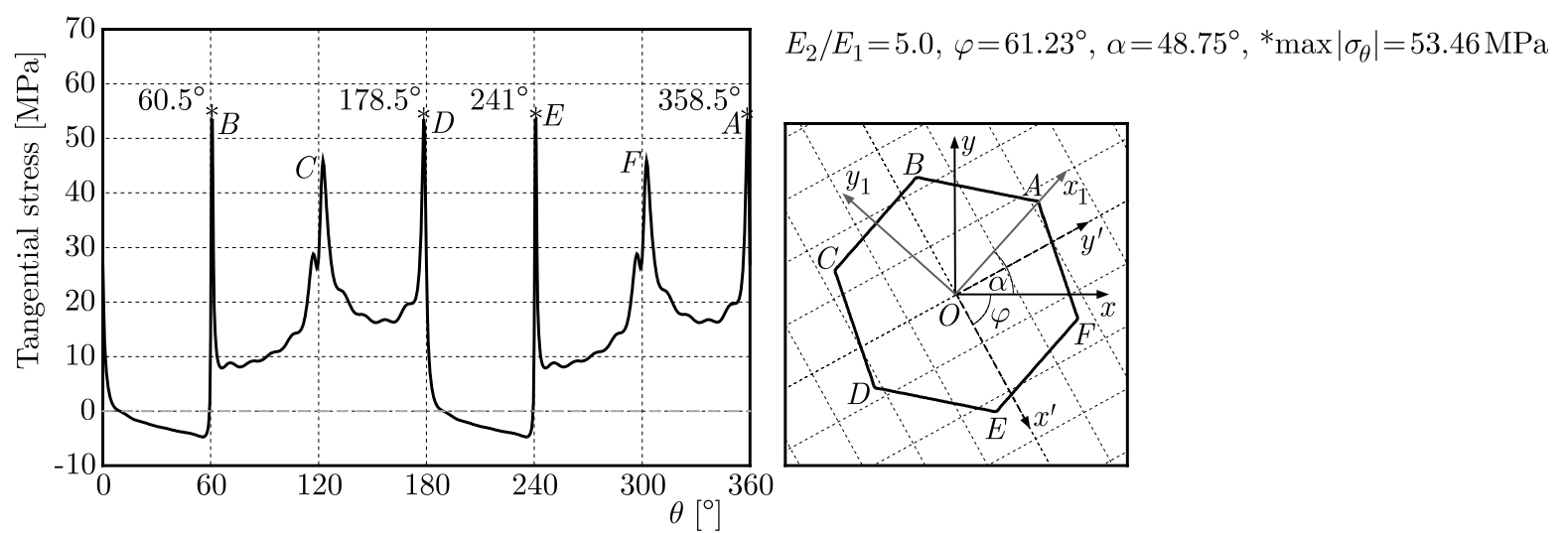

Fig. 13. The optimal placement of fiber and hole orientation angles of orthotropic plates and the tangential stresses along the boundary of the hexagonal hole when $E_{2} / E_{1}=5.0$

-biaxial tensile loading, compressive tangential stress may occur and is more likely to be found in the sides that are parallel ( $B C$ and $E F$ in Figs. 6 and 7), or inclined ( $B C$ and $E F$ in Figs. 11, $A B$ and $D E$ in Figs. 12 and 13$)$ to the direction of the minimum external loading.

Combining with the above example on the hexagonal hole (see Section 3.1.2), we find that for holes with sharp corners, the tangential stress concentration is more likely to occur around the corner points but not always exactly in the corner points. The point $D$ in Fig. 11 gives the most obvious evidence. In addition, the tangential stress concentration is smaller when both the fiber and hole orientation angles are optimized compared to the case in Section 3.1. Thus, when we design a perforated structure in an orthotropic plate, the two parameters should be considered jointly to decrease the tangential stress concentration along the boundary of the hole. From all of the examples given in Sections 3.1 and 3.2, we obtain that for a significantly orthotropic plate $\left(E_{2} / E_{1}=0.2\right.$ or 5.0) with a central hole, setting the principal direction that has the larger Young's modulus inclined to the direction of the minimum loading may decrease the tangential stress concentration along the hole boundary.

\subsubsection{Optimality verification of fiber and hole orientation angles}

The optimality verification should be carried out to guarantee that the optimized fiber and hole orientation angles, $\varphi$ and $\alpha$, are the global optimal solutions. Taking the hexagonal hole as an example, only one condition is analyzed when the ratio of the elastic modulus is given as $E_{2} / E_{1}=2.0$. The external loads and other parameters are the same as in the previous examples. The variation of the maximum tangential stress $\max \left|\sigma_{\theta}\right|$ with respect to $\varphi, \alpha$ and the contour map is illustrated in Fig. 14. The degree intervals of $\varphi$ and $\alpha$ are all set as $0.01^{\circ}$. It can be seen from Fig. 14 that the value of $\max \left|\sigma_{\theta}\right|$ differs for different $\varphi$ and $\alpha$. According to the calculation results, $\max \left|\sigma_{\theta}\right|$ reaches the minimum value of $47.30 \mathrm{MPa}$ only when $\varphi=55.53^{\circ}$ and $\alpha=54.60^{\circ}$, which verifies the results shown in Fig. 12.

\section{Conclusions}

To decrease the tangential stress concentration around an arbitrarily shaped hole in an orthotropic plate, the optimization design on the fiber orientation angle and the hole orientation angle are conducted. The tangential stress distributions around non-circular shapes are given. The results show that the tangential stress concentration around a hole with corner points in an orthotropic plate under an inequi-biaxial loading is more likely to occur in/near the corner points. Furthermore, the tangential stress concentration around a hole with corner points is found to 


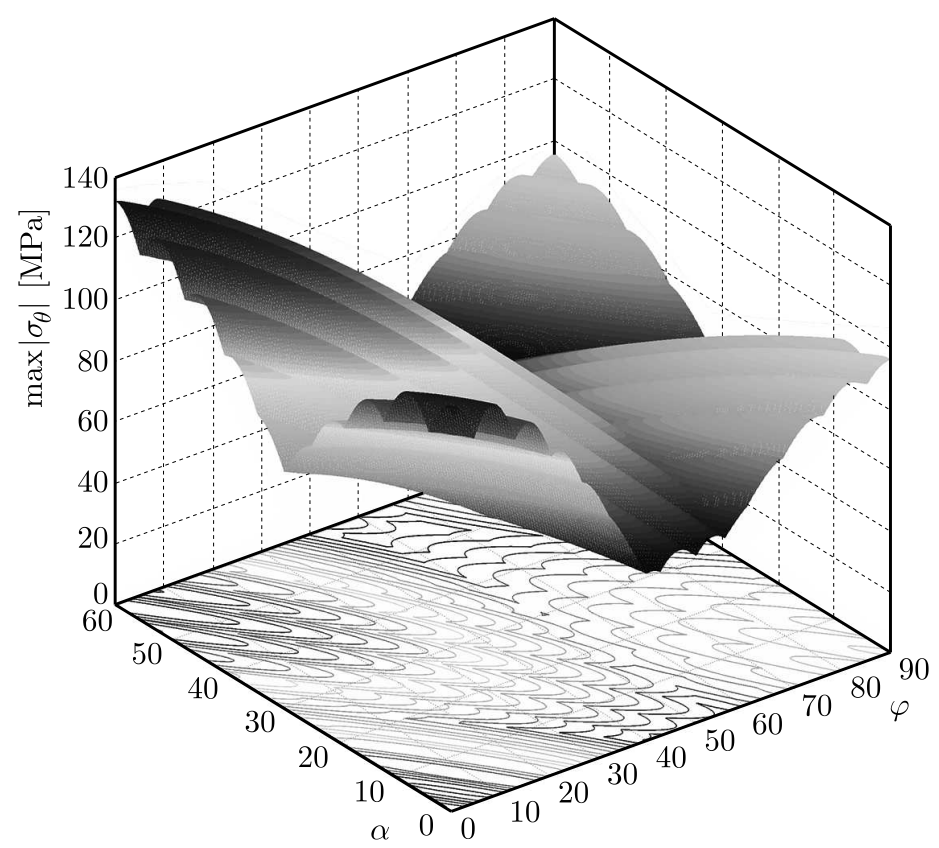

Fig. 14. The three-dimensional map and contour map of $\max \left|\sigma_{\theta}\right|$ for all possible placements of the fiber and hole orientation angles

be more significant compared to that around a smooth convex hole. Under an inequi-biaxial loading, compressive stress can usually be found and is mainly located at the sides that are parallel or inclined to the direction of the minimum external loading.

Based on the results, the following several treatments can be referred in the designing of orthotropic plates in order to decrease the tangential stress concentration: (1) avoid holes with corner points and choose smooth convex holes instead, such as an ellipse hole; (2) set the major axis of an ellipse in the direction of the maximum external loading and (3) set the principal direction that has the larger Young's modulus inclined to the direction of the minimum loading, especially for a significantly orthotropic plate.

Funding

This research work was supported by the Natural Science Foundation of China (Grant No. 11572126, 51704117) and the Fundamental Research Funds for the Central Universities (NCEPU2016XS55).

\section{References}

1. Bjorkman G.S., Richards R., 1976, Harmonic holes - an inverse problem in elasticity, Journal of Applied Mechanics, 43, 3, 414-418

2. Bjorkman G.S., Richards R., 1979, Harmonic holes for nonconstant fields, Journal of Applied Mechanics, 46, 3, 573-576

3. Chen Z.Y., 1994, Analytical Method of Rock Mechanics Analysis, China Coal Industry Publishing House, Beijing

4. Daoust J., HoA S.V., 1991, An analytical solution for anisotropic plates containing triangular holes, Composite Structures, 19, 2, 107-130

5. DhIR S.K., 1981, Optimization in a class of hole shapes in plate structures, Journal of Applied Mechanics, 48, 4, 905-908

6. Engels H., Zakharov D., Becker W., 2001, The plane problem of an elliptically reinforced circular hole in an anisotropic plate or laminate, Archive of Applied Mechanics, 71, 9, 601-612 
7. JAIn N.K., 2009, Analysis of stress concentration and deflection in isotropic and orthotropic rectangular plates with central circular hole under transverse static loading, World Academy of Science Engineering and Technology, 3, 12, 1513-1519

8. Lekhnitskil S.G., 1968, Anisotropic Plates, Gorden and Breach, New York

9. Lekhnitski S.G., 1981, Theory of Elasticity of an Anisotropic Body, Mir Publishers, Moscow

10. Li C., Zheng Y.P., 2007, Influence of different orifice figure on hole-edge stress of composite material plate with hole (in Chinese), Engineering Mechanics, 24, 10, 19-24

11. Lu A.Z., Chen H.Y., Qin Y., Zhang N., 2014a, Shape optimisation of the support section of a tunnel at great depths, Computers and Geotechnics, 61, 3, 190-197

12. Lu A.Z., Chen H.Y., Qin Y., Zhang N., 2014b, Shape optimization of tunnel support section under contact condition of pure slip, Chinese Journal of Rock Mechanics and Engineering, 33, 8, 1563-1571

13. Lu A.Z., Zhang N., Zhang X.L., Lu D.H., Li W.S., 2015, Analytic method of stress analysis for an orthotropic rock mass with an arbitrary-shaped tunnel, International Journal of Geomechanics, 15, 4, 04014068

14. Muskhelishvili N.I., 1963, Some Basic Problems of the Mathematical Theory of Elasticity, Noordhoff, Groningen

15. Rao D.K.N., Babu M.R., Reddy K.R.N., Sunil D., 2010, Stress around square and rectangular cutouts in symmetric laminates, Composite Structures, 92, 12, 2845-2859

16. Ren G., Smith J.V., TAng J.W., XiE Y.M., 2005, Underground excavation shape optimization using an evolutionary procedure, Computers and Geotechnics, 32, 2, 122-132

17. Rezaeepazhand J., Jafari M., 2008, Stress analysis of composite plates with non-circular cutout, Key Engineering Materials, 385-387, 365-368

18. Romeo G., 2001, Analytical and experimental behavior of laminated panels with rectangular opening under biaxial tension, compression and shear loads, Journal of Composite Materials, 35, 8, 639-664

19. Savin G.N., 1961, Stress Concentration Around Holes, Pergamon Press, New York

20. Sharma D.S., 2011, Stress concentration around circular/elliptical/triangular cutouts in infinite composite plate, Proceedings of the World Congress on Engineering, 3, 2626-2631

21. Sharma D.S., Patel N.P., Trivedi R.R., 2014, Optimum design of laminates containing an elliptical hole, International Journal of Mechanical Sciences, 85, 8, 76-87

22. SoвótKa M., ŁydżBA D., RóżAŃski A., 2013, Shape optimization of underground excavation by simulated annealing, Studia Geotechnica et Mechanica, 35, 1, 209-218

23. Storn R., Price K., 1997, Differential evolution - a simple and efficient heuristic for global optimization over continuous spaces, Journal of Global Optimization, 11, 4, 341-359

24. Toubal L., Karama M., Lorrain B., 2005, Stress concentration in a circular hole in composite plate, Composite Structures, 68, 1, 31-36

25. Ukadgaonker V.G., Kakhandki V., 2005, Stress analysis for an orthotropic plate with an irregular shaped hole for different in-plane loading conditions - Part 1, Composite Structures, 70, $3,255-274$

26. Ukadgaonker V.G., Rao D.K.N., 2000, A general solution for stresses around holes in symmetric laminates under in plane loading, Composite Structures, 49, 3, 339-354 